\title{
Plasmonic phase-gradient metasurface for spontaneous emission control
}

\author{
L. Langguth, A. H. Schokker, K. Guo, and A. F. Koenderink* \\ Center for Nanophotonics, FOM Institute AMOLF, Science Park 104, 1098 XG Amsterdam, The Netherlands
}

(Received 21 July 2015; revised manuscript received 2 October 2015; published 2 November 2015)

\begin{abstract}
We combine the concept of phase-gradient metasurfaces with fluorescence directionality control of an ensemble of incoherent emitters. We design a periodic metasurface to control the scattering amplitude of the lattice in momentum space. The lattice is embedded in a waveguiding layer doped with organic fluorophores. In contrast to the usual symmetric directionality that plasmonic lattices impart on emission, we find that the phase gradient translates into asymmetric directional emission into the far field, determined by scattering on a subset of the reciprocal lattice vectors. The measured asymmetry is well explained by analytical modeling.
\end{abstract}

DOI: 10.1103/PhysRevB.92.205401

PACS number(s): 42.25.Fx, 73.20.Mf

\section{INTRODUCTION}

In nano-optics, metal particles are used as antennas for electromagnetic waves in the visible or infrared part of the spectrum [1-3]. They allow localizing fields in much smaller volumes than the diffraction limit [2-4]. As for antennas in the radio frequency and microwave regime, the spectral shape and polarization response of the scattered field of an antenna can be tailored by shape and orientation of the constituent particles. Antennas consisting of multiple elements can exhibit highly directional scattering properties and have been used to direct fluorescence of single local sources or ensembles of incoherently emitting sources in well-defined far-field directions [5-10]. Besides their interest for enhancing directional light emission, antennas in two-dimensional arrays have recently attracted strong interest as so-called metasurfaces. Metasurfaces are able to control the wavefront with a single layer of scatterers [11-15]. A metasurface is formally defined as a 2D planar arrangement of resonant scatterers much thinner then the wavelength, where scatterers may be unequal, and the distance of any scatterer to its neighbors is small compared to the wavelength [16]. To first order, when a metasurface is illuminated by an incident wave, e.g., a plane wave $E_{0} e^{i \mathbf{k} \cdot \mathbf{r}}$, a local polarization is induced of which the amplitude and phase is controlled on a per building block basis by design of each scatterer [16]. For instance, one could imagine a sheet of particles at subwavelength spacing at coordinates $\mathbf{R}_{m}$ that have a polarizability $\alpha_{m}$ where the phase and magnitude of each polarizability tensor is individually optimized. If the particles only weakly couple so that they are mainly driven by the incident field, the amplitude and phase distribution of the polarizability can be directly imprinted as an amplitude and phase distribution on the output beam [17]. A unique feature that is advantageous for applications is that metasurfaces are effectively infinitely thin, whereas they perform functions that are usually solved through bulky secondary optics. For example, the wavefront curvature can be changed, and metasurfaces can thereby form plasmonic lenses [12,14] despite being only $20 \mathrm{~nm}$ thick. The polarization and phase profile of transmitted and reflected beams can be modified to focus beams or to generate beams with orbital angular momentum $[11,13,18]$. All these concepts

\footnotetext{
*Corresponding author: F.Koenderink@amolf.nl
}

apply to far-field properties, i.e., to the transmission, reflection, and scattering of beams, often of well-defined incident wave vectors.

Our aim is to investigate how far the concept of metasurfaces can be applied to spatially incoherent light sources embedded in the metasurface. In the framework of LED-driven solid-state lighting, plasmonic structures have been successfully used to improve the conversion of blue light into longer wavelengths in particular through improvements in pump-field absorption on the one hand and directional outcoupling of light on the other hand $[9,10,19]$. In particular photoluminescence enhancements above 50-fold have been achieved over narrow angular ranges of a few degrees using simple periodic plasmon lattices. Further, highly directional emission has been shown for periodically structured thermal emitters [20]. Here we ask if the concept of plasmonic metasurfaces can be fruitfully applied to ensembles of incoherent local sources, such as fluorophores in light conversion layers.

This paper is structured in the following way. First we choose a metasurface design which is compatible with fluorescence experiments. Next, we present measured polarizationresolved fluorescence radiation patterns for the designed metasurface and a reference structure. Finally we discuss the markedly asymmetric beaming with a simple single-scattering model neglecting particle-particle interactions and also in a full coupled dipole calculation taking multiple scattering and polarization into account.

\section{STRUCTURE/DESIGN STRATEGY}

In order to test the metasurface principle with local fluorescent sources, an antenna array compatible with the geometry and wavelength used in typical fluorescence experiments is required. We choose the geometry recently studied by Rodriguez et al. [9,19] and by Schokker et al. [21], which consists of antennas residing on a glass substrate (Menzel Gläser, $n_{\text {glass }}=1.52$ ) covered in a 400-nm-thick layer of a high-index polymer (SU8). Due to its high refractive index $\left(n_{\text {SU8 }}=1.65\right)$, the photoresist acts as a single mode slab waveguide. The vertical geometry is shown in Fig. 1(a), while Fig. 1(b) shows a top-view scanning electron micrograph. Fluorescent Rhodamine 6G (Rh6G) molecules mixed in the polymer act as an ensemble of randomly oriented and randomly distributed dipole sources, with a fluorescence emission band at 550-600 $\mathrm{nm}$. In previous experiments $[9,10,21,22]$ 


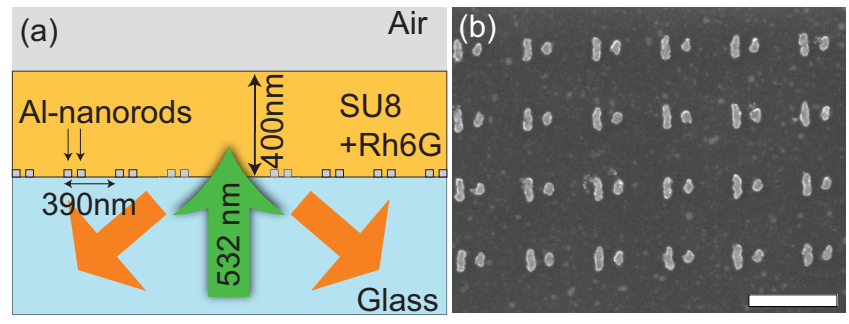

FIG. 1. (Color online) (a) Schematic cross section of the sample: An aluminum antenna array is located on a glass substrate and embedded in a 400-nm-thick high-index polymer layer which is doped with Rh6G molecules. Excitation of the fluorophores is performed by a pulsed $532 \mathrm{~nm}$ laser though an oil-immersion objective at the glass side. Fluorescence emission is collected through the same objective. (b) SEM micrograph of the antenna array taken after liftoff before spin coating the polymer layer (scale bar: $500 \mathrm{~nm}$ ). Dimension $[l \times w \times t]$ : long antenna $[130 \times 40 \times 30] \mathrm{nm}^{3}$; short antenna $[70 \times 40 \times 30] \mathrm{nm}^{3}$; antenna distance (center to center) $d=120 \mathrm{~nm}$; pitch: $390 \mathrm{~nm}$.

directional photoluminescence enhancement in the normal direction was achieved for square lattices of single plasmonic scatterers of pitch $380-400 \mathrm{~nm}$. As the distance corresponds closely to the wavelength in the waveguide, scattering of the waveguide mode at the periodic antennas leads to emission preferably normal to the sample surface.

We want to tailor a unit cell which provides a phase gradient for scattered light and thus acts as directional antenna. The resonance in a particle imposes a phase shift on scattering varying from 0 to $180^{\circ}$ when the resonance frequency is swept from the blue side to the red side of the emission wavelength. The resonance frequency of plasmons can be tuned by size [12,14], shape [11], or material [23]. As building blocks for our metasurface we choose metal rods of different lengths. To gain phase contrast we require metal particles which exhibit a resonance around the fluorescence emission wavelength when they are embedded in the high-index dielectric. The resonance wavelength of standard plasmonic particles (gold and silver) of $\approx 50 \mathrm{~nm}$ size or above are already at longer wavelength than the desired $580 \mathrm{~nm}$ and would further shift to longer wavelength when being embedded in the polymer. To obtain a resonant response at the desired wavelength, we hence have to use aluminium, which provides a higher plasma frequency than gold or silver.

The scattered field amplitude and phase of aluminum rods of different lengths is simulated with a commercial finite element Maxwell solver, COMSOL Multiphysics 4.4b. For all simulations and calculations we use the dielectric function of aluminum given by Rakić et al. in Ref. [24]. Simulations are performed in the frequency domain and contain a single aluminum $\operatorname{rod}\left[l \times w \times t=[50-150] \times 40 \times 30 \mathrm{~nm}^{3}\right.$, sketch in Fig. 2(a)] on the surface of a semi-infinite glass substrate, with its long axis aligned along the $y$ axis. A $y$-polarized (along the long axis of the rod) plane wave is used as excitation field, incident from the glass side (amplitude: $1 \mathrm{~V} / \mathrm{m}$ ), taking the Fresnel reflection at the glass-SU8 interface into account. We run the calculation for rod lengths from $l=50-150 \mathrm{~nm}$ in $10 \mathrm{~nm}$ steps. For each combination of geometry and frequency the current density averaged over the wire volume is extracted.

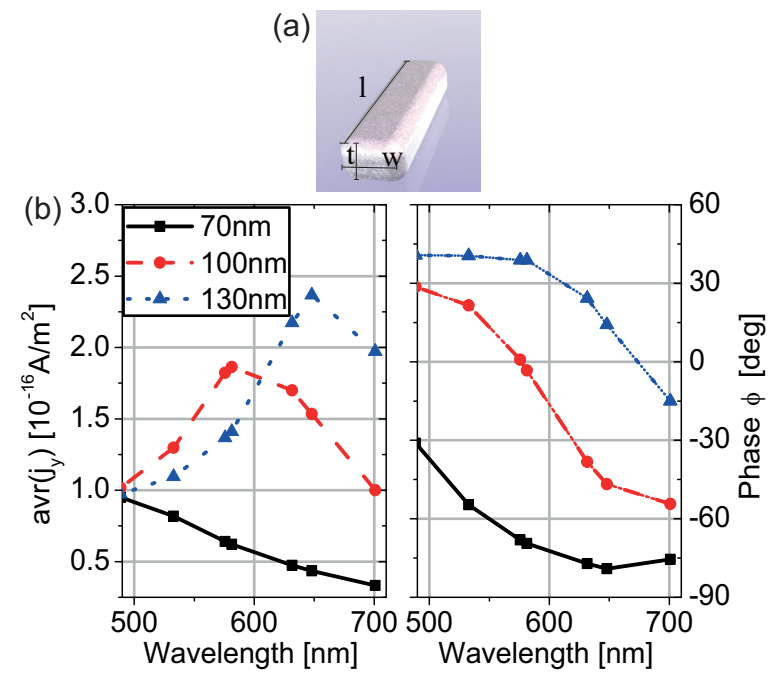

FIG. 2. (Color online) (a) Schematic of the single rod antenna analyzed with the finite element Maxwell solver (Comsol Multiphysics $4.4 \mathrm{~b}$ ). The rod of length $l$, width $w$, and thickness $t$ is placed on glass $\left(n_{\text {glass }}=1.52\right)$ in high index resist SU8 $\left(n_{\text {SU8 }}=1.65\right)$. (b) Volume-averaged current density (left) and phase lag to the driving field (right) of the volume-averaged current density along the long axis in three rods of length $l=70 \mathrm{~nm}$ (solid black line, squares), $100 \mathrm{~nm}$ (dashed red line, circles), and $130 \mathrm{~nm}$ (dotted blue line, triangles) length.

The volume-averaged current density is an oscillating quantity, the amplitude of which is plotted in Fig. 2(b), while Fig. 2(c) shows the phase relative to the incident field. The current peaks at the resonance, when most energy is stored in the resonator, at which point the phase goes through a large change [Fig. 2(c)].

Figure 2(b) shows the averaged current and the relative phase for three wire lengths: 70, 100, and $130 \mathrm{~nm}$. While the resonance of the $130 \mathrm{~nm}$ wire (blue) is at approximately $650 \mathrm{~nm}$ wavelength and the shortest wire has its peak at a wavelength smaller than $500 \mathrm{~nm}$, the $100 \mathrm{~nm}$ wire has its resonance at our design wavelength of $580 \mathrm{~nm}$. Additionally, because of the strong phase difference of about $100^{\circ}$ between the scattered field of the $130 \mathrm{~nm}$ and the $70 \mathrm{~nm}$ wire, we choose these two elements as the building block to form a simple two element phase gradient antenna. We set the particle distance to $120 \mathrm{~nm}$ [see Fig. 3(a)]. This value is chosen to sufficiently break the left right symmetry of the lattice and can be reproducibly fabricated with electron beam lithography.

To determine the directional properties of this dimer antenna, we performed full wave simulations and obtained radiation patterns from a near- to far-field transformation [25]. To apply the Stratton-Chu transformation, one requires that the entire scatterer is placed in a homogeneous medium. Therefore the antenna is entirely immersed in the high-index resist $(n=1.65)$ and the glass interface is ignored in this calculation. The scattered power [shown in Fig. 3(b)] in the $-x$ direction is more than twice the power scattered in the $+x$ direction for a driving field with a wave vector aligned along the $z$ axis and polarization along the long axis of the rods. This two element phase-gradient unit cell hence acts a directional scatterer, which scatters preferentially into the direction of the long rod. 

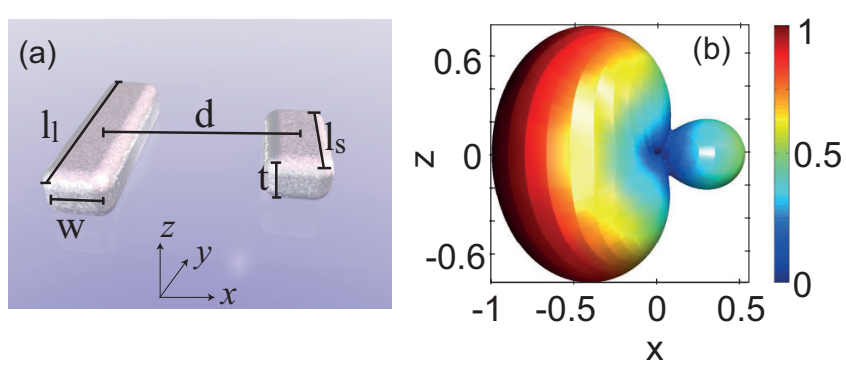

FIG. 3. (Color online) (a) Simulated phase gradient antenna: The centers of both rods are aligned on the $x$ axis. The long rod $(130 \mathrm{~nm})$ is centered at $x=-60 \mathrm{~nm}$ and the short rod one $(70 \mathrm{~nm})$ at $x=$ $60 \mathrm{~nm}$. (b) Radiation pattern of the scattered field of the rod pair in a homogeneous medium of 1.65 . The rods are excited with equal phases by a plane wave with a wave vector aligned along the $z$ axis. The radiation pattern is normalized to its maximum value, which occurs asymmetrically, in the direction where the long rod is located. In the positive direction, a factor 2 less power is radiated than in the negative $x$ direction.

\section{SAMPLE FABRICATION AND SET UP}

To study the collective emission from the dye distributed in the plane, we arranged the antennas in a $2 \mathrm{D}$ square array with a pitch of $390 \mathrm{~nm}$ and $100 \times 100 \mu \mathrm{m}^{2}$ in size. Figure 1(b) shows a SEM micrograph of a phase-gradient antenna array fabricated with electron beam lithography in a positive resist (ZEP520) on a glass cover slide with the conditions given in Ref. [21]. Exposure was done using dot exposure with doses of $(1-1.5) \times 10^{-4} \mathrm{pC}$ using an electron gun voltage of $20 \mathrm{kV}$ and a current of $0.11 \mathrm{nA}$. After exposure we developed the resist in pentyl acetate, a $1: 3$ methyl isobutyl ketone/isopropyl alcohol mixture. Aluminium was deposited via electron beam evaporation at a pressure of $\approx 1 \times 10^{-6} \mathrm{mbar}$ with an evaporation rate of $2 \AA /$ s. Liftoff was performed in anisole at $50{ }^{\circ} \mathrm{C}$. On the same samples we also fabricated reference structures with only one rod per unit cell instead of a dimer. To fabricate the waveguide with fluorophores on top of the antenna array, we use the negative photoresist SU8 and dope it with Rh6G by dissolving $5.25 \mathrm{mg}$ of Rh6G perchlorate in $1 \mathrm{~mL}$ of cyclopentanone. The cyclopentanone with Rh6G is added to $1 \mathrm{ml}$ of SU8-2005, after which we ultrasonicate the solution for $10 \mathrm{~min}$. The solution has a Rh6G perchlorate concentration of $0.25 \mathrm{wt}$. \%. Spin coating the SU8 solution at $2000 \mathrm{rpm}$ on the antenna array samples results in a $\approx 400 \mathrm{~nm}$ thick SU8 layer. Finally, the samples were baked for $2 \mathrm{~min}$ at $90{ }^{\circ} \mathrm{C}$ to evaporate remaining cyclopentanone.

Our measurements are performed in a home-built fluorescence Fourier microscope with laser excitation (Teem Photonics, type STG-03E-1S0) at $532 \mathrm{~nm}$ wavelength. A detailed description of the used setup is given by Schokker et al. in Ref. [21]. Figure 4 shows a schematic of the microscope as configured for "back focal plane" imaging. The center of the antenna array is illuminated through an oilimmersion objective (Nikon Plan Apo $\lambda 100 \times / 1.45$ NA) with the excitation hitting the sample plane as a collimated beam, with an approximate diameter of $50 \mu \mathrm{m}$. The excitation is pulsed ( $\approx 500$ ps duration) and has an excitation energy density per pulse of about $5 \mathrm{~mJ} / \mathrm{cm}^{2}$, far below any saturation level
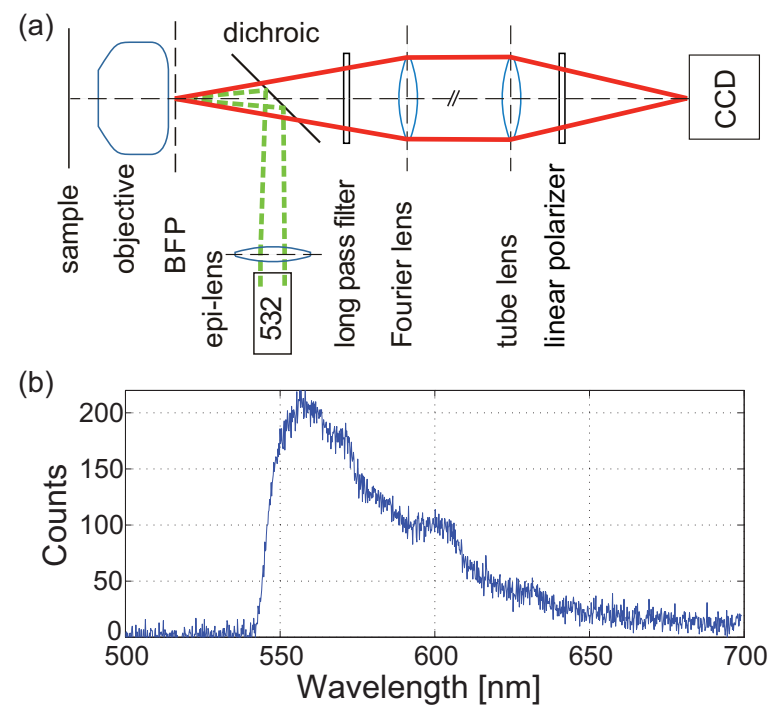

FIG. 4. (Color online) (a) Schematic of the used Fourier fluorescence microscope. The pulsed excitation laser (green dashed line) is focused on the back focal plane (BFP) to give a collimated beam in the sample plane. The fluorescence emission (red solid line) is picked up by the same objective, and the BFP is imaged by a Fourier lens and a tube lens on a CCD. The green excitation light is suppressed by a long-pass filter. (b) Fluorescence emission spectrum of Rhodamine $6 \mathrm{G}$ in the SU8 resist layer, as measured after the long-pass filter.

for this dye system. The fluorescence is collected through the same objective and passes a dichroic beamsplitter (Semrock, Di01-R532-25x36) and a long pass filter (Chroma, HHQ545lp) to block the excitation light.

After the spectral filtering, a polarization is selected with a linear polarizer (Thorlabs LPVIS100), and the back-focal plane (BFP) of the objective is imaged via a Fourier lens [26] on a thermoelectrically cooled Si CCD (Andor CLARA). By this we measure the angular distribution of the fluorescence emission for vertical and horizontal polarization.

\section{MEASUREMENT}

Figure 5 shows angle-resolved fluorescence images of two different structures each at polarization perpendicular [panels (b) and (e)] and parallel [panels (c) and (f)] to the long axis of the rods. Each plot corresponds to a sum over 10 excitation pulses. Each fluorescence image is a back focal plane image, meaning that images appear as a bright disk of fluorescence with radius set by the maximum collection angle of the microscope objective. Since the NA is 1.45 , this corresponds to emission at parallel wave vectors $k_{\|} / k_{0}=$ 1.45 where $k_{0}=\omega / c$, or equivalently emission angles into the glass of $\arcsin (1.45 / 1.52)=72^{\circ}$. The center of each image corresponds to fluorescence propagating along the optical axis, normal to the sample surface. The spatial coordinate in the images directly corresponds to parallel momentum. Besides the outer ring at the objective NA, a steplike increase of fluorescence intensity is evident at $k_{\|}=k_{0}$, which is caused by the fact that fluorescence at an air-dielectric interface is preferentially emitted at angles just above NA $=1$, as shown in Refs. [27,28]. 

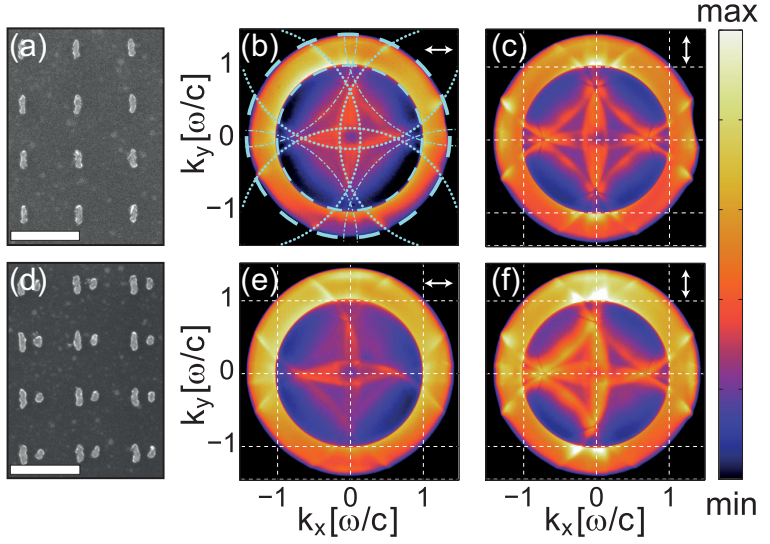

FIG. 5. (Color online) SEM micrographs of two samples: panel (a) a single rod per unit cell, panel (d) phase gradient dimer array (scale bars: $500 \mathrm{~nm}$ ). Angular fluorescence intensity distributions of the single rod array [panels (b),(c)] and the dimer array [panels (e),(f)] for polarization aligned perpendicular [panels (b),(e)] and parallel [panels (c),(f)] to the rods. Excitation powers $\approx 0.13 \mu \mathrm{J}$ per pulse for the single rod array and $\approx 0.23 \mu \mathrm{J}$ per pulse for the dimer array. In panel (b) we have indicated the following guides to the eye for reading panels (b),(c),(e),(f): (i) dashed circles concentric with the origin indicate NA $=1$ (inner) and the microscope NA (outer ring), (ii) waveguide dispersion (radius of curvature 1.57 times that of NA = 1 ring) diffracted by $2 \pi / a( \pm 1,0)$ and $2 \pi / a(0, \pm 1)$ indicated by dotted lines, and (iii) dispersion diffracted by $2 \pi / a( \pm 1, \pm 1)$ indicated by dash-dotted lines. We list the minima and maxima of the color scaling in each image: For the single rod array, $x$-pol. [1800-2800], $y$-pol. [3500-8000], and for the dimer array, $x$-pol. [4000-8000], y-pol. [4000-8000], where numbers refer to CCD pixel counts.

Multiple intersecting circles of high intensity appear both in measurements of the phase gradient dimer antenna array as well as for the reference structure of the single rod array. For both structures the circles have the highest visibility in the polarization aligned along the long axis of the rods [Figs. 5(c) and 5(f)], which matches our expectation that the rods scatter the strongest when polarized along their long axis. Measurements of the perpendicular polarization are shown for completeness, but we will focus the discussion on the parallel polarization.

We explain the generic features in the measurements by discussing the reference structure (single rods) in vertical polarization, shown in Fig. 5(c). There are eight intersecting circles forming a cross shape. A mode index of $k_{\mathrm{WG}}=1.57 k_{0}$ at the $580 \mathrm{~nm}$ is obtained when calculating the dispersion relation for our wave-guide geometry [Fig. 6(c)] by using the formula given in Eqs. (4.4) and (4.17) of Ref. [29]. The radius of curvature of the circular features in our measurement indeed corresponds well to $1.57 k_{0}$. Therefore we conclude that the Fourier images represent an isofrequency cut through the repeated zone scheme waveguide dispersion relation. The periodic lattice provides reciprocal lattice vectors at which photons in the waveguide can scatter. The reciprocal lattice vectors relevant in our measurement are located at $G=$ $\frac{2 \pi}{a} \cdot[n ; m]$ with $n ; m=-1,0,1$ and the pitch $a=390 \mathrm{~nm}$. Copies of the waveguide mode circle appear centered around each reciprocal lattice vector. The circle centered around the
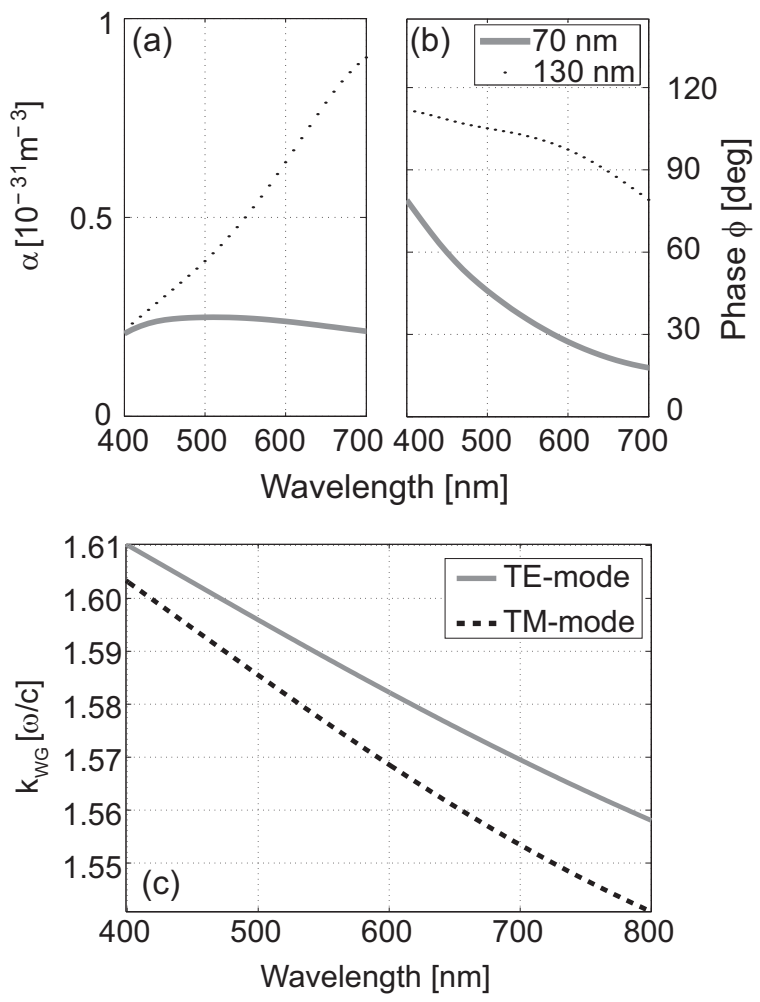

FIG. 6. (a) Absolute value $|\alpha|$ and (b) phase $\phi=\arg (\alpha)$ of the polarizability tensor along the long axis of rods of $70 \mathrm{~nm}$ (gray solid lines) and $130 \mathrm{~nm}$ (black dotted lines). The phase shows a $90^{\circ}$ offset compared to Fig. 2 due to the fact that, in a time-harmonic driving field, the polarization of a dipole and its point current are $90^{\circ}$ out of phase. (c) Relevant dispersion relations in dielectric slab: TE (gray solid line) and TM (black dashed line) waveguide mode.

$\Gamma$ point $\left(\left[k_{x} ; k_{y}\right]=[0 ; 0]\right)$ corresponds to the bare waveguide mode and has a parallel wave vector greater than the light line in glass. Hence it is not visible in our measurement. The eight circles intersecting from reciprocal lattice vectors of nonzero momentum are blurred due to spectral averaging over the fluorescence bandwidth of $\approx 50 \mathrm{~nm}$.

For the single element unit cell, circles originating at $\frac{2 \pi}{a}[0 ; \pm 1]$ and $\frac{2 \pi}{a}[ \pm 1 ; 0]$ intersect close to the $\Gamma$ point, which means that in our experiment the waveguide mode has a slightly higher momentum than $2 \pi / a$. In other words, the experiment is slightly detuned from the second order Bragg diffraction condition [30]. Furthermore there are four circles originating from the diagonals $\frac{2 \pi}{a}[ \pm 1 ; \pm 1]$ intersecting on the $x$ or $y$ axis at approximately $\frac{3}{4} k_{0}$. The radiation pattern possesses the symmetries of the reference structure, i.e., two mirror planes along the $x$ and $y$ axis.

Now we turn to a discussion of the radiation pattern of the phase-gradient antenna array. Again intersecting circles are observed. However, the three circles originating at $k_{x}=2 \pi / a$ and $k_{y}=[-1 ; 0 ; 1] \cdot 2 \pi / a$ show hugely reduced intensity. The radiation pattern, as well as the structure, does not possess a mirror symmetry around the $y$ axis anymore. Introducing a phase gradient in the unit cell has translated into a selective asymmetric scattering at a subset of reciprocal lattice vectors. This shows that even for an incoherent ensemble of local 
sources, the concept of phase-gradient metasurfaces can be employed to shape the angular distribution of photons.

\section{MODELING}

\section{A. First order model}

To show how the directional scattering properties of each unit cell translate to an asymmetry in the band intensities measured in the far field, we use a classical model from radio wave antenna theory [17]. The model assumes that radiation into the far field originates from the induced polarization in the antennas, which is given by the product of the local driving field $A_{n}$ and a scalar polarizability $\alpha$ of each particle. In this scalar model the vector nature of the electric field is neglected and $A$ is assumed to be a scalar complex-valued driving field. The radiated field in a viewing direction parameterized by unit vector $\hat{k}$ pointing into the glass reads

$$
E=\frac{e^{i k R}}{R} S(\hat{k}) \sum_{n \in \text { lattice }} \alpha_{n} A_{n} e^{-i \mathbf{k} \cdot \mathbf{r}_{n}}
$$

with $r_{n}$ the position of the scatterer, $A_{n}$ the complex amplitude of the driving field, $\alpha_{n}$ the scalar polarizability of the particle, $S$ the radiation pattern of the single element, and $\mathbf{k}=$ $\frac{\omega}{c} n_{\text {glass }} \hat{k}$. The scattered field factorizes in the single element radiation pattern $S(\hat{k})$ and a factor determined by the array geometry [17]. This is analogous to solid-state physics where an $\mathrm{X}$-ray diffraction pattern can be factorized in a structure and form factor [31].

For each antenna element we calculate the polarizability tensor in the waveguide environment using a method explained in the following section. Our scalar model only uses the polarizability along the long axis of the rods, a constraint that is relaxed later when we perform coupled dipole calculations. Figure 6 shows the absolute value $|\alpha|$ (a) and phase $\phi$ (b) of the polarizabilities of the long (black dotted lines) and short (gray solid lines) rod. The spectral behavior of this polarizability matches well with that of induced current in the FEM simulations (compare to Fig. 2).

We decompose our antenna array into a two-dimensional lattice of Dirac- $\delta \mathrm{s}$, representing the periodic lattice (denoted as $\Delta$ ) and two Dirac- $\delta$ s, separated by a distance $d$, with different complex amplitudes mimicking the response of antennas in the unit cell: $U C(\mathbf{r})=\alpha_{l} \delta\left(-\frac{d}{2}\right)+\alpha_{s} \delta\left(\frac{d}{2}\right)$. The convolution $\Delta * U C$ yields the phase gradient antenna array, as illustrated in the two left pictograms in the top of Fig. 7. Having defined the geometry, we turn to describing the driving field. In the experiment our system is driven by an incoherent ensemble of point sources emitting in a fluorescence band around $580 \mathrm{~nm}$ $(516 \mathrm{THz})$. To capture the essential physics, we take as driving source frequency $516 \mathrm{THz}$ and describe the analysis for a single point source located at the origin. Different source positions can be treated similarly using the Fourier shift theorem, as discussed at the end of this section. The point source radiates a cylindrical wave of wave number $k_{\mathrm{WG}}=1.57 k_{0}$ into the waveguide, that acts as driving for the phase-gradient antennas. Mathematically, the driving field for the antenna array reads $A_{w g}(\mathbf{r})=e^{i|\mathbf{r}| \cdot k_{\mathrm{WG}}} \cdot A_{0} \cdot|\mathbf{r}|^{-1 / 2}$.

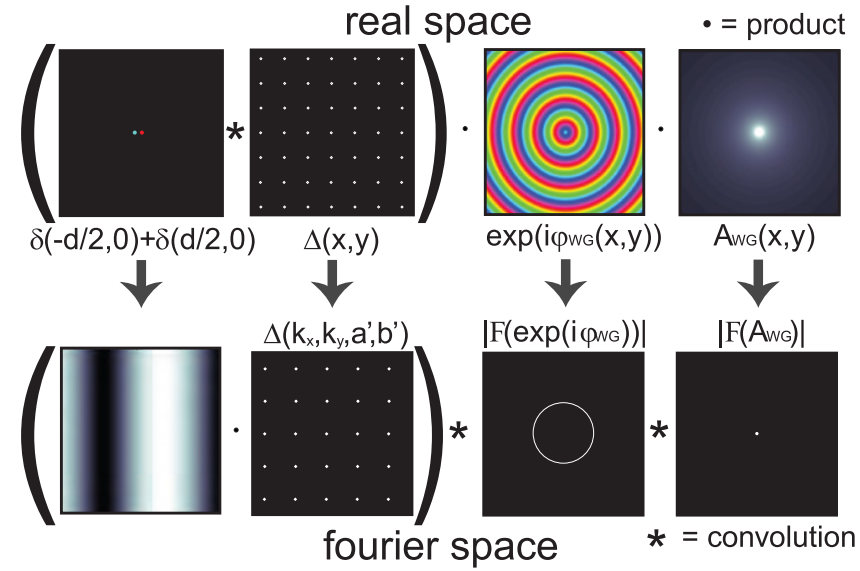

FIG. 7. (Color online) Illustration of the real space factorization and the respective Fourier transform. The brightness of a pixel indicates amplitude; the color value indicates the phase. Top row depicts the real space representation of the phase-gradient antenna array, constructed via the convolution of the rod pair (left) of the single antenna with a periodic lattice of Dirac- $\delta$ s. The constructed antenna array is multiplied with the driving field of a point source in the center with a radial phase profile (second from right) and a decaying amplitude (right). The bottom row shows the corresponding Fourier transforms.

Returning to the infinite sum

$$
\sum_{n \in \text { lattice }} \alpha_{n} A_{n} e^{-i \mathbf{k} \cdot \mathbf{r}_{n}},
$$

we now realize that the source distribution that generates the far field is the product of the antenna array geometry with the local complex valued driving field

$$
J(\mathbf{r})=[U C(\mathbf{r}) * \Delta(\mathbf{r}, \mathrm{a})] \cdot A_{w g}(\mathbf{r})
$$

as illustrated in the top row of Fig. 7. The field radiated into the far field is given by the Fourier transform $(\mathcal{F} \mathcal{T})$ of the source distribution [17]

$$
E\left(k_{x}, k_{y}\right) \propto \mathcal{F} \mathcal{T}[J(\mathbf{r})]=\int J(\mathbf{r}) \cdot e^{-i \mathbf{k} \cdot \mathbf{r}} d^{3} \mathbf{r}
$$

that is easily obtained by calculating the Fourier transform of each term in the factorization and using the convolution theorem. The real space configuration and the respective $\mathcal{F} \mathcal{T}$ s are illustrated in Fig. 7. $J(\mathbf{r})$ is a product of two factors, the lattice geometry, and the driving field, with each of the factors constructed either by a convolution or a product. First we look at the $\mathcal{F} \mathcal{T}$ of the geometry, constructed as the convolution of the lattice with the unit cell. We call its Fourier transform "antenna array factor" $\mathcal{A}(\mathbf{k})$ to indicate that it combines the "antenna factor" [in X-ray diffraction language known as "form factor," the Fourier transform of $U C(\mathbf{r})]$ and the "array factor" [in X-ray diffraction known as "structure factor," Fourier transform of the periodic lattice $\Delta(\mathbf{r}, a)$ ]. Fourier transforming the unit cell gives a slowly varying but asymmetric sinusoidal amplitude modulation. Transforming the periodic square lattice with pitch $a$ yields the reciprocal lattice with the same symmetry and a pitch of $2 \pi / a$. According to the convolution theorem, the FT of a product of two functions is given by the convolution of their $\mathcal{F} \mathcal{T}$ s: $\mathcal{F} \mathcal{T}[G \cdot H]=\mathcal{F} \mathcal{T}[G] * \mathcal{F} \mathcal{T}[H]$, 
and vice versa: $\mathcal{F} \mathcal{T}[G * H]=\mathcal{F} \mathcal{T}[G] \cdot \mathcal{F} \mathcal{T}[H]$. Thus, the antenna-array factor, i.e., the $\mathcal{F} \mathcal{T}$ of the geometry is given by the product of the two $\mathcal{F} \mathcal{T}$ s and yields a square lattice multiplied with the $\mathcal{F} \mathcal{T}$ of the unit cell. The positions of the peaks are given by the square lattice, and their complex amplitude is given by the unit cell [32].

We proceed with transforming the driving field $\mathcal{F} \mathcal{T}\left[\left|A_{\mathrm{WG}}\right| \cdot \exp (i \varphi)\right]$, which is given by the $\mathcal{F} \mathcal{T}$ of the phase factor convoluted with the $\mathcal{F} \mathcal{T}$ of the amplitude factor. The phase map yields an isofrequency cut through the waveguide dispersion relation, which is a circle with radius $k_{\mathrm{WG}}=1.57 k_{0}$. The $\mathcal{F} \mathcal{T}$ of the amplitude function is a peak of finite size but with a full width at half maximum much smaller than $\left|k_{w g}\right|$, meaning that the sharp circle is blurred through convolution [33]. We call the Fourier transform of the source distribution "driving factor" $\mathcal{D}(\mathbf{k})$, for lack of a better term.

The full radiation pattern can now be constructed as a convolution of the reciprocal lattice with the blurred circle of the waveguide mode [Fig. 8(a)]. This results in copies of the WG circles centered around each reciprocal lattice vector, multiplied with the respective complex amplitude, as we show in Fig. 8. The $\Gamma$ point in the center of the graph is enclosed by four intersecting circles. The circle centered around $\left[k_{x}, k_{y}\right]=[-2 \pi / a, 0]$ shows an intensity $\approx 3 \times$ higher than the circle centered around $[+2 \pi / a, 0]$. Already this strongly simplified model captures the key features of the experiment and explains well the asymmetry of the radiation pattern. Our simple geometry of two scatterers with a phase gradient shows control over the relative scattering strength of left- and right-pointing reciprocal lattice vectors. Taking multiple scatterers with phase and amplitude in one unit cell and using this simple design rule allows us to enhance or suppress scattering at selected reciprocal lattice vectors.

At this point, we have rationalized why in classical antenna theory a single point source at the lattice origin will give a radiation pattern consistent with our measurement. In our experiment, however, we deal with the incoherent sum of signals that results from many single molecules that emit single photons at a time and are randomly dispersed over the unit cell. First, it is important to note that for a single emitter that is fixed in position, the classical antenna calculation describes the angular probability amplitude distribution for each emitted photon [1]. Second, to obtain the incoherent sum over many emission events from many molecules spread uniformly through the system, one needs to incoherently average the intensities resulting from all possible point source positions. Extending our analysis to obtain the radiation pattern for a single point source at any arbitrary position shifted from the origin by $\Delta r$ is trivial, since by the Fourier shift theorem this introduces a phase factor $e^{i \mathbf{k} \cdot \Delta \mathbf{r}}$ in the driving factor, so that the radiation pattern will be modified to

$$
E\left(k_{x}, k_{y} ; \Delta \mathbf{r}\right) \propto \mathcal{A}(\mathbf{k}) *\left[e^{i \mathbf{k} \cdot \Delta \mathbf{r}} \mathcal{D}(\mathbf{k})\right] .
$$

From this result, one can easily derive the incoherent sum, integrating $\left|E\left(k_{x}, k_{y} ; \Delta \mathbf{r}\right)\right|^{2}$ over all source positions $\Delta \mathbf{r}$

$$
I_{\text {incoh }}\left(k_{x}, k_{y}\right)=\int_{\text {entire unit cell }}\left|E\left(k_{x}, k_{y} ; \Delta \mathbf{r}\right)\right|^{2} d \Delta r .
$$

The result is shown in Fig. 8(b). Compared to the single-source position calculation [Fig. 8(a), $\left|E\left(k_{x}, k_{y} ; \Delta \mathbf{r}=0\right)\right|^{2}$ ] two main
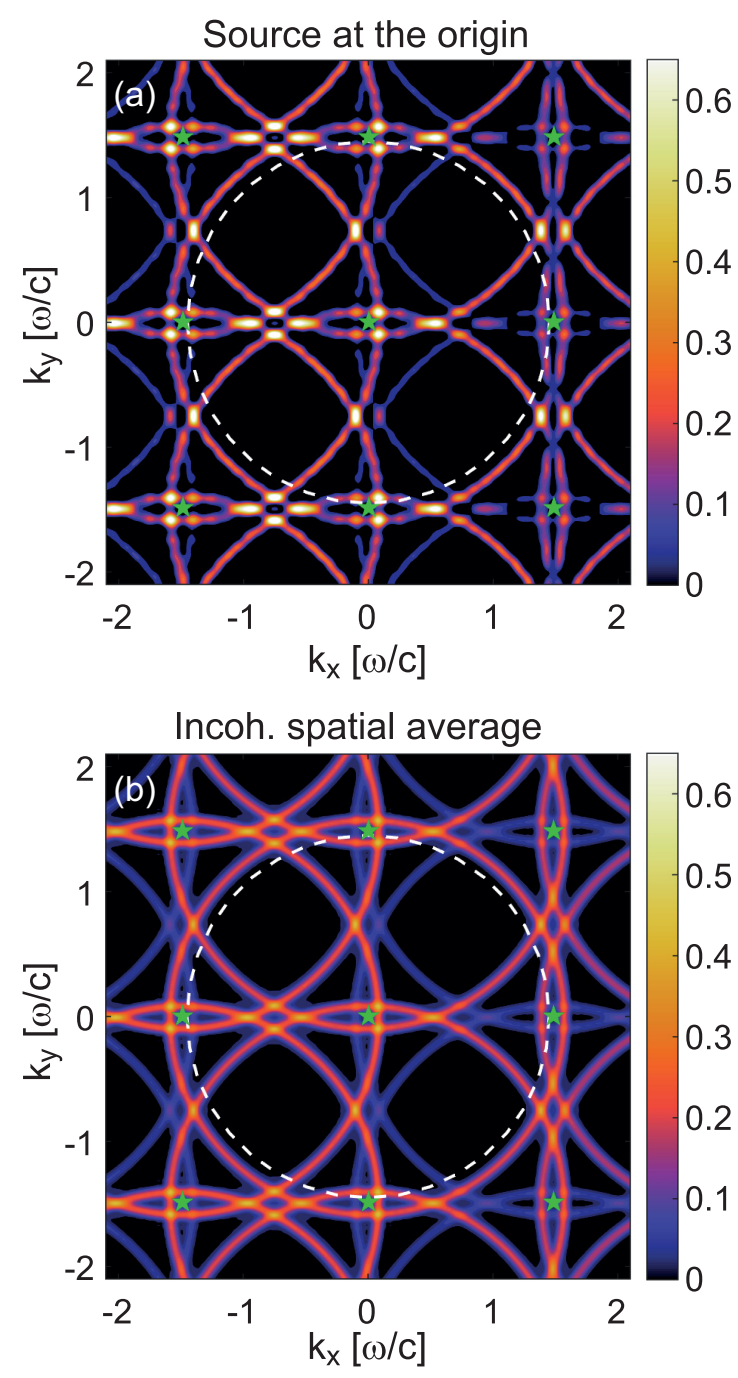

FIG. 8. (Color online) (a) Numerical calculation of the radiation pattern by convolution of the waveguide dispersion relation (circles) with the reciprocal lattice (green pentagrams) for a single source placed at the origin. The intensity is normalized to its maximum. The white dashed circle indicates the maximum collection angle of $1.45 k_{0}$ in the experiment. (b) Incoherent ensemble radiation pattern obtained by averaging over the entire unit cell as per Eq. (3). This pattern is normalized to twice its maximum for direct comparison with panel (a): the brightest spots in (a) at circle crossings are due to interference and hence twice as strong as the incoherent average.

features stand out. First, the radiation pattern again consists of repeated-zone scheme rings with a similarly asymmetric intensity distribution. This shows that the simple model indeed captures the main physics behind our observations. Second, the incoherent average shows smoother circles, and the large intensity variations near crossings of circles are strongly reduced. Both these features can be easily understood from Eq. (23). Since both $\mathcal{A}(\mathbf{k})$ and $\mathcal{D}(\mathbf{k})$ are sparse (being a set of points, resp. a circle), for all source positions the field in Eq. (2) is negligible except in the set of circles. The relative phase of these circles depends on $\Delta r$. When calculating intensity, the phase is irrelevant whenever just a single circle contributes. However, at intersections of circles interferences occur, leading to the dark and bright fringes near circle 
crossings, evident in Fig. 8(a). Upon incoherent averaging these interference fringes average out. To conclude, our singlesource analysis is robust against incoherent averaging. We warn the reader that for general structures and driving profiles, incoherent averages can differ markedly from a coherent single-point source calculation.

\section{B. Coupled dipole model}

To check how the simplified scalar single scattering model compares to an electrodynamic model that contains polarization, retardation, and the wave guide physics, we use a coupled dipole calculation. This is the method of choice since the problem of a single source above a periodic lattice is not amenable to full-wave analysis with finite element or finite difference solvers with periodic boundary conditions, given that periodic boundary conditions replicate not just scatterers but also sources. Tackling the problem of a single source in a periodic system could be done with a full wave solver using the reciprocity theorem [1], and combining many plane-wave-incidence calculations that can be done with periodic boundary conditions. However, this approach would require sampling of order $>10^{4}$ incidence angles for a single structure and wavelength to match experiment, each of which would require approximately 100 seconds on a $2.4 \mathrm{GHz}$ Xeon multicore computer, yielding an impractical computation time.

Compared to the well-known coupled dipole method (CDM) for particles in free space [34,35], we account for the waveguide geometry by using the Green's function for the air/polymer/glass system [28,29]. As is customary in the CDM each antenna is characterized by a polarizability tensor $\alpha$. We now take the full tensorial character of the polarizability of the scatterers into account and calculate the polarizability tensor. Therefore we first evaluate the static polarizability with the depolarization matrix of a rectangular volume [36] and the permittivity of aluminum, embedded in a background medium with the refractive index of SU8. We then add radiation damping to the polarizability, which we calculate by evaluating the imaginary part of the Green's function at the location of the scatterer in the layered medium $[29,37]$. This provides us with a dynamic polarizability which is input for electrodynamically self-consistent multiple scattering calculations.

In an array of $N$ particles, the dipole moment induced in particle $m$ is due to the total field at the particle position, which is the superposition of the driving field $E_{\text {inc }}$ and the field scattered from all the other particles. This leads to a set of equations,

$$
\mathbf{p}_{m}=\underline{\underline{\alpha}}\left[\mathbf{E}_{\mathrm{inc}}+\sum_{n \neq m} \mathbf{G}\left(\mathbf{r}_{m}, \mathbf{r}_{n}\right) \mathbf{p}_{n}\right],
$$

which can be solved self-consistently to obtain the dipole moments $\mathbf{p}_{m}$ of each antenna. The interaction between dipoles of different position is given by the Green's function $\mathbf{G}\left(\mathbf{r}_{m}, \mathbf{r}_{n}\right)$ of our waveguide geometry [Chap. 10 of Ref. [28]]. Including in total 1922 scatterers in our finite-array calculation, the modeled array covers a square area of approximately $12 \mu \mathrm{m} \times$ $12 \mu \mathrm{m}$.

As driving field we place a dipole source within the waveguide in the unit cell in the center of the array and solve
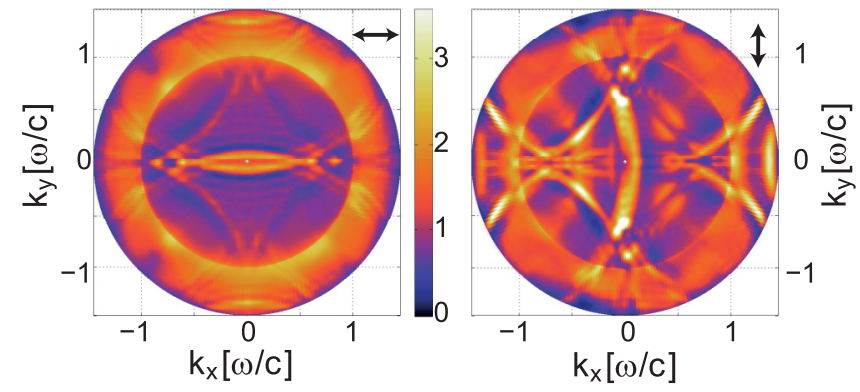

FIG. 9. (Color online) Radiation patterns of the phase gradient antenna array for horizontal (left) and vertical (right) polarization, calculated with a coupled dipole model for dipoles inside the waveguide. Both figures share the same color map. As in the measurement (Fig. 5) we plot the intensity distribution in the back focal plane multiplied by $\cos (\theta)$.

for the induced dipole moments in each antenna. We then calculate the scattering into the far field, using the asymptotic far-field Green's function and transform the angular emission into the back focal plane image according to the Abbe sine condition [Chap. 3 of Ref. [28]]. By that we obtain the full electric field, including polarization, in the BFP as they are then mapped in our experiment on our CCD. We calculate the far-field image for three orthogonal source dipole orientations at eight different positions inside the unit-cell volume in the waveguide. Finally we sum up the far-field intensities for each polarization channel of all the 24 calculations of different source orientations and positions. The results of the calculation are shown in Fig. 9. Comparing to the measurements shown in Fig. 5, we find good agreement and find all the key characteristics well reproduced by the CDM. In horizontal polarization, perpendicular to the rod orientation, only minor left-right asymmetry is observed. In contrast, in $y$ polarization, there is strong intensity from the waveguide modes scattered at $k_{x}=-2 \pi / a$ and almost no intensity in those waveguide modes scattered at $k_{x}=2 \pi / a$.

The coupled dipole model also allows us to evaluate the near field in the waveguide. An interesting question is whether the directional scattering properties of the unit cell only translate into directional outcoupling or also into an asymmetric population of the waveguide mode. To answer this question we calculate the near field in the middle of the waveguide, $200 \mathrm{~nm}$ away from both the glass and air interface. We perform a 2D Fourier transform for every field component for every source orientation at each of the eight source positions. We then sum the power spectrum for all polarizations for all source orientations and positions. The result is displayed in Fig. 10, showing the parallel wave-vector content of the fields inside the waveguide, plotted in a logarithmic color scale spanning three orders of magnitude in wave-vector intensity. We see that the highest amplitudes are in the waveguide mode centered around the $\Gamma$ point, confirming our intuition that the system is weakly scattering and justifying the assumption of the simplified model in the previous section. Around the circle perimeter we find only a minor modulation of the amplitude, with minima whenever a diffraction condition is met. For wave vectors $k_{\|}<\omega / c$, the maximum intensity is more than a factor 100 smaller than in the waveguide mode. Only a very 


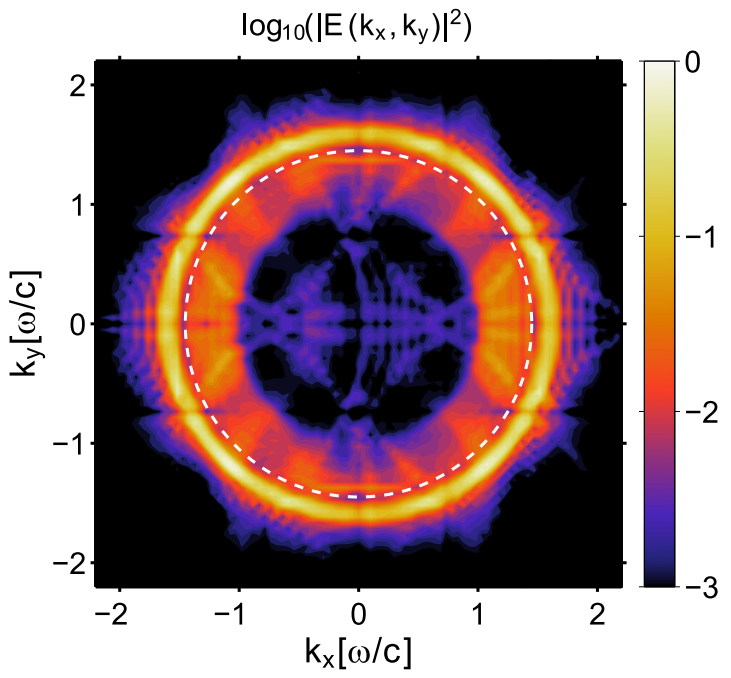

FIG. 10. (Color online) Angular power spectrum of the Fourier transform of the electric field in the waveguide. In large part the amplitude is located in the unscattered waveguide mode. The white dashed line indicates the maximum collection angle of the objective at $1.45 k_{0}$. The intensity is normalized to the maximum; the color scale is logarithmic spanning three orders of magnitude.

minor left-right asymmetry in population of the mode inside the waveguide is visible, in contrast to the asymmetry of the scattering out into the far field.

\section{CONCLUSION/OUTLOOK}

We have shown that the concept of a phase-gradient unit cell to build a metasurface allows us to shape and transform the farfield emission pattern of ensembles of randomly positioned, incoherent emitters, e.g., fluorophores. This finding adds to the observations made earlier that coupling incoherent sources to a guided mode provides a high degree of spatial coherence within the guided mode and thus allows us to control the angular distribution of the light scattering into the far field by diffractive plasmonic lattice resonances [20]. Phase gradients built in the unit cell allow us to control the scattering amplitude on different reciprocal lattice vectors and thus asymmetric photoluminescence enhancement.

In our paper we made use of the fact that a planar waveguide, that is only just sufficiently thin to be single mode, has good overlap with the particles, and also of the fact that the slow $1 / \sqrt{r}$ field decay in a waveguide ensures many unit cells are significantly polarized [21,38]. Similar asymmetries can occur in other planar waveguiding systems that abide by the same rules, such as slots in $\mathrm{Au}$ or Ag films, provided the slots have an engineered phase and amplitude response [39]. Beyond the case of waveguides, we expect the pronounced asymmetries to also extend to the case of "surface lattice resonances," i.e., long-range collective lattice modes that form in the absence of a vertical index contrast and are mediated by grating anomalies [40]. Recently, waveguide systems and surface lattice resonance systems have attracted significant attention for the possibility of semiclassical strong coupling between the photonic/plasmonic resonances and the resonant polarizabilities of high concentrations of embedded dye $[19,41]$. It would be interesting to study how this collective effect of strong coupling evolves as asymmetry in the unit cell is introduced. We note that as a precursor of such collective coherent phenomena, we have observed lasing operation at various band crossings in the phase-gradient array system at pump levels similar to those reported by Schokker [21].

\section{ACKNOWLEDGMENTS}

This work is part of the research program of the Foundation for Fundamental Research on Matter (FOM), which was financially supported by The Netherlands Organization for Scientific Research (NWO). This work was furthermore supported by NanoNextNL, a microtechnology and nanotechnology consortium of the Government of the Netherlands and 130 partners.
[1] P. Bharadwaj, B. Deutsch, and L. Novotny, Adv. Opt. Photonics 1, 438 (2009).

[2] L. Novotny and N. van Hulst, Nat. Photonics 5, 83 (2011).

[3] V. Giannini, A. I. Fernández-Domínguez, S. C. Heck, and S. A. Maier, Chem. Rev. 111, 3888 (2011).

[4] D. K. Gramotnev and S. I. Bozhevolnyi, Nat. Photonics 4, 83 (2010).

[5] T. H. Taminiau, F. D. Stefani, F. B. Segerink, and N. F. van Hulst, Nat. Photonics 2, 234 (2008).

[6] A. G. Curto, G. Volpe, T. H. Taminiau, M. P. Kreuzer, R. Quidant, and N. F. van Hulst, Science 329, 930 (2010).

[7] D. Dregely, R. Taubert, J. Dorfmüller, R. Vogelgesang, K. Kern, and H. Giessen, Nat. Commun. 2, 267 (2011).

[8] L. Langguth, D. Punj, J. Wenger, and A. F. Koenderink, ACS Nano 7, 8840 (2013).

[9] S. R. K. Rodriguez, S. Murai, M. A. Verschuuren, and J. G. Rivas, Phys. Rev. Lett. 109, 166803 (2012).
[10] G. Lozano, D. J. Louwers, S. R. Rodríguez, S. Murai, O. T. Jansen, M. A. Verschuuren, and J. Gómez Rivas, Light Sci. Appl. 2, e66 (2013).

[11] N. Yu, P. Genevet, M. A. Kats, F. Aieta, J.-P. Tetienne, F. Capasso, and Z. Gaburro, Science 334, 333 (2011).

[12] H. Gao, J. K. Hyun, M. H. Lee, J.-C. Yang, L. J. Lauhon, and T. W. Odom, Nano Lett. 10, 4111 (2010).

[13] G. M. Lerman and U. Levy, Opt. Lett. 33, 2782 (2008).

[14] L. Lin, X. M. Goh, L. P. McGuinness, and A. Roberts, Nano Lett. 10, 1936 (2010).

[15] X. Ni, N. K. Emani, A. V. Kildishev, A. Boltasseva, and V. M. Shalaev, Science 335, 427 (2012).

[16] N. Yu and F. Capasso, Nat. Mater 13, 139 (2014).

[17] C. A. Balanis, Antenna Theory: Analysis and Design, 3rd ed. (Wiley, New York, 2012).

[18] N. Mohammadi Estakhri and A. Alù, Phys. Rev. B 89, 235419 (2014).

[19] S. Rodriguez and J. G. Rivas, Opt. Express 21, 27411 (2013). 
[20] C. Arnold, F. Marquier, M. Garin, F. Pardo, S. Collin, N. Bardou, J.-L. Pelouard, and J.-J. Greffet, Phys. Rev. B 86, 035316 (2012).

[21] A. H. Schokker and A. F. Koenderink, Phys. Rev. B 90, 155452 (2014).

[22] S. Murai, M. A. Verschuuren, G. Lozano, G. Pirruccio, S. R. K. Rodriguez, and J. G. Rivas, Opt. Express 21, 4250 (2013).

[23] T. Shegai, S. Chen, V. D. Miljković, G. Zengin, P. Johansson, and M. Käll, Nat. Commun. 2, 481 (2011).

[24] A. D. Rakic, A. B. Djurisic, J. M. Elazar, and M. L. Majewski, Appl. Opt. 37, 5271 (1998).

[25] J. Stratton and L. Chu, Phys. Rev. 56, 99 (1939).

[26] C. M. Dodson, J. A. Kurvits, D. Li, and R. Zia, Opt. Lett. 39, 3927 (2014).

[27] M. A. Lieb, J. M. Zavislan, and L. Novotny, J. Opt. Soc. Am. B 21, 1210 (2004)

[28] L. Novotny and B. Hecht, Principles of Nano-Optics (Cambridge University Press, Cambridge, UK, 2006).

[29] H. P. Urbach and G. L. J. A. Rikken, Phys. Rev. A 57, 3913 (1998).

[30] H. Rigneault and F. Lemarchand, Opt. Lett. 24, 148 (1999).
[31] C. Kittel, Introduction to Solid State Physics, 8th ed. (Wiley, New York, 2005), p. 703.

[32] S. Larouche and D. R. Smith, Opt. Lett. 37, 2391 (2012).

[33] Mathematically, the real space expression $e^{i|\mathbf{r}| \cdot k_{\mathrm{WG}}} \cdot A_{0} \cdot|\mathbf{r}|^{-1 / 2}$ is approximate. The field of a point source in the $2 \mathrm{D}$ scalar wave equation with complex propagation constant is exactly given in the Fourier domain as $\left|\mathbf{k}^{2}-k_{\mathrm{WG}}^{2}\right|^{-1}$. The waveguide dispersion appears as pole.

[34] B. T. Draine and P. J. Flatau, J. Opt. Soc. Am. A 11, 1491 (1994),

[35] F. J. G. de Abajo, Rev. Mod. Phys. 79, 1267 (2007).

[36] L. Langguth, Coupling in Metamaterials, Diplomarbeit, Universität Stuttgart, 2010.

[37] F. Bernal Arango, A. Kwadrin, and A. F. Koenderink, ACS Nano 6, 10156 (2012).

[38] S. R. K. Rodriguez, Y. T. Chen, T. P. Steinbusch, M. A Verschuuren, A. F. Koenderink, and J. G. Rivas, Phys. Rev. B 90, 235406 (2014).

[39] G. Li, M. Kang, S. Chen, S. Zhang, E. Y.-B. Pun, K. W. Cheah, and J. Li, Nano Lett. 13, 4148 (2013).

[40] S. R. K. Rodriguez, A. Abass, B. Maes, O. T. A. Janssen, G. Vecchi, and J. Gómez Rivas, Phys. Rev. X 1, 021019 (2011).

[41] P. Törmä and W. L. Barnes, Rep. Prog. Phys. 78, 013901 (2015). 